\title{
On termination of meta-programs
}

\author{
Alexander Serebrenik, Danny De Schreye \\ Department of Computer Science, K.U. Leuven \\ Celestijnenlaan 200A, B-3001, Heverlee, Belgium \\ Email: \{Alexander.Serebrenik, Danny.DeSchreye\}@cs.kuleuven.ac.be
}

\section{Introduction}

The term meta-programming refers to the ability of writing programs that have other programs as data and exploit their semantics [4]. The choice of logic programming as a basis for meta-programming offers a number of practical and theoretical advantages. One of them is the possibility of tackling critical foundation problems of meta-programming within a framework with a strong theoretical basis. Another is the surprising ease of programming. These reasons motivated an intensive research on metaprogramming inside the logic programming community $[4,16,19,22,23]$.

On the other hand, termination analysis is one of the most intensive research areas in logic programming as well. See [12] for the survey. More recent work on this topic can be found among others in $[14,18,20,24,30]$.

Traditionally, termination analysis of logic programs have been done either by the "transformational" approach or by the "direct" one. A transformational approach first transforms the logic program into an "equivalent" term-rewrite system (or, in some cases, into an equivalent functional program). Here, equivalence means that, at the very least, the termination of the term-rewrite system should imply the termination of the logic program, for some predefined collection of queries ${ }^{1}$. Direct approaches do not include such a transformation, but prove the termination directly on the basis of the logic program. In [25] we have developed an approach that provides the best of both worlds: a means to incorporate into "direct" approaches the generality of general term-orderings.

The aim of this paper is presenting a methodology allowing us to perform a correct termination analysis for a broad class of meta-interpreters together with different classes of object programs. Unlike the previous

\footnotetext{
${ }^{1}$ The approach of Arts [5] is exceptional in the sense that the termination of the logic program is concluded from a weaker property of single-redex normalisation of the term-rewrite system.
} 
work on compositionality of termination proofs [3] the approach presented allows a simple reuse of the termination proof of the object program for the meta-program.

This methodology is based on the "combined" approach to termination analysis mentioned above.

Example 1. Our research has been motivated by the famous "vanilla" meta-interpreter $M_{0}$, undoubtly belonging to logic programming classics.

$$
\begin{aligned}
& \text { solve }(\text { true }) \\
& \text { solve }((\text { Atom, Atoms })) \leftarrow \text { solve }(\text { Atom }), \text { solve }(\text { Atoms }) . \\
& \text { solve }(\text { Head }) \leftarrow \text { clause }(\text { Head, Body }), \text { solve }(\text { Body }) .
\end{aligned}
$$

Termination of this meta-interpreter, presented in Example 1, has been studied by Pedreschi and Ruggieri. They proved, that LD-termination of the goal $G$ with respect to a program $P$ implies LD-termination of the goal solve $(G)$ with respect to $M_{0}$ and $P$ (Corollary 40, [23]). However, we claim more: the two statements are equivalent, i.e., the goal $G$ LDterminates with respect to a program $P$ if and only if the goal solve $(G)$ LD-terminates with respect to $M_{0}$ and $P$.

In order for meta-interpreters to be useful in applications they should be able to cope with a richer language than the one of the "vanilla" metainterpreter, including, for example, negation. Moreover, typical applications of meta-interpreters, such as debuggers, will also require producing some additional output or performing some additional tasks during the execution, such as constructing proof trees or cutting "unlikely" branches for an uncertainty reasoner with cutoff. These extensions can and usually will influence termination properties of the meta-interpreter.

By extending the suggested technique [25] to normal programs, we are able to perform the correct analysis of a number of (possibly extended) meta-interpreters, performing tasks as described above. We identify popular classes of meta-interpreters, such as extended meta-interpreters [22], and using this technique prove that termination is usually improved. We also state more generic conditions implying preservation of termination.

The rest of this paper is organised as following. We start by some preliminary remarks and basic definitions. Then, we present the methodology developed applied to the "vanilla" meta-interpreter. Afterwards we show how the same methodology can be applied for more advanced meta-interpreters and conclude. 


\section{Preliminaries}

A quasi-ordering over a set $S$ is a reflexive and transitive relation $\geq$ defined on elements of $S$. If neither $s \geq t$, nor $t \geq s$ we write $s \| t$. An ordered set $S$ is said to be well-founded if there are no infinite descending sequences $s_{1}>s_{2}>\ldots$ of elements of $S$. If the set $S$ is clear from the context we will say that the ordering, defined on it, is well-founded.

We follow the standard notation for terms and atoms. A query is a finite sequence of atoms. Given an atom $A, \operatorname{rel}(A)$ denotes the predicate occurring in $A$. Term $_{P}$ and Atom $_{P}$ denote, respectively, sets of all terms and atoms that can be constructed from the language underlying $P$. The extended Herbrand Universe $U_{P}^{E}$ (the extended Herbrand base $B_{P}^{E}$ ) is a quotient set of $\operatorname{Term}_{P}\left(\right.$ Atom $\left._{P}\right)$ modulo the variant relation.

We refer to an SLD-tree constructed using the left-to-right selection rule of Prolog, as an LD-tree. We will say that a goal $G L D$-terminates for a program $P$, if the $\mathrm{LD}$-tree for $(P, G)$ is finite.

The following definition is borrowed from [1].

Definition 1. Let $P$ be a program and $p, q$ be predicates occurring in it.

- We say that $p$ refers to $q$ in $P$ if there is a clause in $P$ that uses $p$ in its head and $q$ in its body.

- We say that $p$ depends on $q$ in $P$ and write $p \sqsupseteq q$, if $(p, q)$ is in the transitive, reflexive closure of the relation refers to.

- We say that $p$ and $q$ are mutually recursive and write $p \simeq q$, if $p \sqsupseteq q$ and $q \sqsupseteq p$.

We also abbreviate $p \sqsupseteq q, q \nsupseteq p$ by $p \sqsupset q$.

Results for termination of meta-interpreters presented in this paper are based on notion of order-acceptability, introduced in [25]. This notion of order-acceptability generalises the notion of acceptability with respect to a set [13] in two ways: 1) it generalises it to general term orderings, 2) it generalises it to mutual recursion, using the standard notion of mutual recursion [1] - the original definition of acceptability required decrease only for calls to the predicate that appears in the head of the clause. This restriction limited the approach to programs only with direct recursion.

Before introducing the order-acceptability we need the following notion.

Definition 2. [13] Let $P$ be a definite program and $S$ be a set of atomic queries. The call set, $C a l l(P, S)$, is the set of all atoms $A$, such that a variant of $A$ is a selected atom in some derivation for $P \cup\{\leftarrow Q\}$, for some $Q \in S$ and under the left-to-right selection rule. 
Definition 3. Let $S$ be a set of atomic queries and $P$ a definite program. $P$ is order-acceptable with respect to $S$ if there exists a well-founded ordering $>$, such that

- for any $A \in \operatorname{Call}(P, S)$

- for any clause $A^{\prime} \leftarrow B_{1}, \ldots, B_{n}$ in $P$, such that $\operatorname{mgu}\left(A, A^{\prime}\right)=\theta$ exists,

- for any atom $B_{i}$, such that $\operatorname{rel}\left(B_{i}\right) \simeq \operatorname{rel}(A)$

- for any computed answer substitution $\sigma$ for $\leftarrow\left(B_{1}, \ldots, B_{i-1}\right) \theta$ :

$$
A>B_{i} \theta \sigma
$$

In [25] we prove the following theorem.

Theorem 1. Let $P$ be a program. $P$ is order-acceptable with respect to a set of atomic queries $S$ if and only if $P$ is LD-terminating for all queries in $S$.

We discovered in [25] that order-acceptability is a powerful technique, able to analyse a wide variety of programs, such as normalisation [14], derivative [15], bid [10], and credit evaluation expert system [28] to mention a few. In this paper we will see that order-acceptability plays a key role in analysing termination of meta-programs.

\section{Basic definitions}

In this section we present a number of basic definitions. We start by defining the kind of program we call a meta-program. Then we introduce two semantic notions that relate computed answers of the interpreted program and of the meta-program and conclude by discussing an appropriate notion of termination for meta-interpreters.

\subsection{Interpreted programs and meta-programs}

In this subsection we define the notion of a meta-interpreter, that is a program having another program as data. We've seen already in Example 1 that the input program is represented as a set of atoms of the predicate clause. We call this representation a clause-encoding and define it formally as following.

Definition 4. Let $P$ be a program. The clause-encoding $\gamma_{c e}(P)$ is a collection of facts of a new predicate clause, such that clause $(H, B) \in \gamma_{c e}(P)$ if and only if $H \leftarrow B$ is a clause in $P$. 
Example 2. Let $P$ be the following program:

$$
p(a) . \quad p(X) \leftarrow q(X) . \quad q(b) .
$$

Then, the following program is $\gamma_{c e}(P)$ :

$$
\text { clause }(p(a), \text { true } \quad \text { clause }(p(X), q(X)) . \quad \text { clause }(q(b), \text { true }) .
$$

A meta-interpreter for a language is an interpreter for the language written in the language itself. We follow [28] by using a predicate solve for the meta-interpreter.

Definition 5. The program $P$ is called a meta-program if it can be represented as $M \cup I$, such that:

- $M$ defines a predicate solve that does not appear in $I$.

- I is a clause-encoding of some program $P^{\prime}$.

$M$ is called the meta-interpreter. $P^{\prime}$ is called the interpreted program.

We also assume that , $/ 2$ and clause/2 do not appear in the language underlying the interpreted program. Observe, that if this assumption is violated, clear distinction between the meta-interpreter and the interpreted program is no longer possible.

\subsection{Soundness and completeness of meta-interpreters}

Now we are going to define the notions of soundness and completeness for meta-interpreters, that will relate computed answers of the interpreted program to the computed answers of the meta-program.

Definition 6. The meta-interpreter $M$ is called sound if for every program $P$, every goal $G_{0} \in B_{P}^{E}$ and every $H_{1}, \ldots, H_{n} \in U_{M}^{E}$

- if solve $\left(t_{0}, t_{1}, \ldots, t_{n}\right)$ is a computed answer for some $\left\{\operatorname{solve}\left(G_{0}, H_{1}, \ldots, H_{n}\right)\right\} \cup M \cup \gamma_{c e}(P)$

- then $t_{0}$ is a computed answer for $\left\{G_{0}\right\} \cup P$.

Note that $H_{1}, \ldots, H_{n}$ are extra arguments of solve that support added functionality (see Example 3 below). The definition of soundness, as well as further definitions require some property to hold for all programs. Correctness of these definitions does not depend on the class of the programs considered. However, constructing meta-interpreters that will satisfy the properties required for all Prolog programs can be difficult. Thus, we start by restricting the class of programs considered to definite logic programs. In Section 6 we study a broader class of programs. 
Definition 7. The meta-interpreter $M$ is called complete if

- for every program $P$ and every goal $G_{0} \in B_{P}^{E}$

- if $t_{0}$ is a computed answer for $\left\{G_{0}\right\} \cup P$, then

- for every $H_{1}, \ldots, H_{n} \in U_{M}^{E}$

- if there exist $s_{1}, \ldots, s_{n}$ such that solve $\left(s_{0}, s_{1}, \ldots, s_{n}\right)$ is a computed answer for $\left\{\right.$ solve $\left.\left(G_{0}, H_{1}, \ldots, H_{n}\right)\right\} \cup M \cup \gamma_{c e}(P)$

- then there exist $t_{1}, \ldots, t_{n}$ such that solve $\left(t_{0}, t_{1}, \ldots, t_{n}\right)$ is a computed answer for $\left\{\operatorname{solve}\left(G_{0}, H_{1}, \ldots, H_{n}\right)\right\} \cup M \cup \gamma_{c e}(P)$.

Example 3. The following meta-interpreter $M_{1}$ is both sound and complete: $\operatorname{solve}(A) \leftarrow$ fail. It is sound, since there are no computed answers for $\leftarrow \operatorname{solve}(G)$. It is also complete, since $s_{1}, \ldots, s_{n}$ required in Definition 7 do not exist. The "vanilla" meta-interpreter $M_{0}$ (Example 1) is also both sound and complete, as shown by Levi and Ramundo in [19].

The following meta-interpreter $M_{2}$, mimicking the LD-refutation with bounded depth (the depth provided as the second argument) is sound, but is not complete.

$$
\begin{aligned}
& \text { solve }(\text { true, } 0) . \\
& \operatorname{solve}((A, B), N) \leftarrow \operatorname{solve}(A, N), \operatorname{solve}(B, N) . \\
& \operatorname{solve}(A, s(N)) \leftarrow \operatorname{clause}(A, B), \operatorname{solve}(B, N) .
\end{aligned}
$$

It is intuitively clear why this meta-interpreter is sound. To see that it is not complete, let $P$ be a program resented in Example 2, let $G_{0}$ be $p(X)$ and let $t_{0}$ be $p(b)$. Then, given $H_{1}=s(0)$ there exist $s_{1}$, such that solve $\left(s_{0}, s_{1}\right)$ is a computed answer for $\left\{\operatorname{solve}\left(G_{0}, H_{1}\right)\right\} \cup M \cup \gamma_{c e}(P)$. Namely, $s_{1}=s(0)$ and $s_{0}=p(a)$. However, there exists no $t_{1}$ such that $\operatorname{solve}\left(p(b), t_{1}\right)$ is a computed answer for $\left\{\operatorname{solve}\left(G_{0}, H_{1}\right)\right\} \cup M \cup \gamma_{c e}(P)$.

\subsection{Notion of termination}

Recall that our aim is to study termination of meta-interpreters, that is termination of goals of the form solve $\left(G_{0}, H_{1}, \ldots, H_{n}\right)$, where $G_{0}$ is a goal with respect to the interpreted program. Thus, the crucial issue is defining an appropriate notion of termination for meta-interpreters.

For many applications, such as debuggers, the desired behaviour of a meta-interpreter is to preserve termination. However, there are many meta-interpreters that may change termination behaviour of the interpreted program, either by improving or by violating it.

Definition 8. (non-violating LD-termination) 
1. Let $M$ be a meta-interpreter defining solve with arity 1. $M$ is called non-violating LD-termination if for every program $P$ and every goal $G_{0} \in B_{P}^{E}$ if the LD-tree of $\left\{G_{0}\right\} \cup P$ is finite, then the LD-tree of $\left\{\right.$ solve $\left.\left(G_{0}\right)\right\} \cup\left(M \cup \gamma_{c e}(P)\right)$ is finite as well.

2. Let $M$ be a meta-interpreter defining solve with arity $n+1, n>0$. $M$ is called non-violating LD-termination with respect to $S \subseteq\left(U_{M}^{E}\right)^{n}$ if for every program $P$ and every goal $G_{0} \in B_{P}^{E}$ if the LD-tree of $\left\{G_{0}\right\} \cup P$ is finite, then for every sequence $\left(H_{1}, \ldots, H_{n}\right) \in S$, the $L D$-tree of $\left\{\right.$ solve $\left.\left(G_{0}, H_{1}, \ldots, H_{n}\right)\right\} \cup\left(M \cup \gamma_{c e}(P)\right)$ is finite as well.

It should be noted, that traditionally this feature is called improving termination. However, this name is not quite successful, since by improving we do not mean that the meta-program terminates more often than the original one, but that it terminates at least as often as the original one. Thus, we chose to use more clear names.

It also follows from the definition of non-violation that every metainterpreter defining solve with arity greater than 1 does not violate termination with respect to the empty set.

Example 4. Recall once more the meta-interpreters shown in Example 3. $M_{1}$ does not violate termination, and $M_{2}$ does not violate termination with respect to $\left(U_{M_{2}}^{E}\right)^{1}$, that is with respect to $U_{M_{2}}^{E}$.

Definition 9. (non-improving LD-termination)

1. Let $M$ be a meta-interpreter defining solve with arity 1. $M$ is called non-improving LD-termination if for every program $P$ and every goal solve $\left(G_{0}\right) \in B_{M \cup \gamma_{c e}(P)}^{E}$, finiteness of the LD-tree of $\left\{\right.$ solve $\left.\left(G_{0}\right)\right\} \cup(M \cup$ $\left.\gamma_{c e}(P)\right)$ implies finiteness of the LD-tree of $\left\{G_{0}\right\} \cup P$.

2. Let $M$ be a meta-interpreter defining solve with arity $n+1, n>$ 0. $M$ is called non-improving LD-termination with respect to $S \subseteq$ $\left(U_{M}^{E}\right)^{n}$ if for every program $P$ and every goal solve $\left(G_{0}, H_{1}, \ldots, H_{n}\right) \in$ $B_{M \cup \gamma_{c e}(P)}^{E}$, such that $\left(H_{1}, \ldots, H_{n}\right) \in S$, finiteness of the LD-tree of $\left\{\operatorname{solve}\left(G_{0}, H_{1}, \ldots, H_{n}\right)\right\} \cup\left(M \cup \gamma_{c e}(P)\right)$ implies finiteness of the $L D$ tree of $\left\{G_{0}\right\} \cup P$.

Example 5. The meta-interpreter $M_{1}$ improves termination, while the meta-interpreter $M_{2}$ does not improve it w.r.t Vars, where Vars is a set of variables.

Finally, we will say that a meta-interpreter $M$ defining solve with arity $n+1$ is preserving termination (preserving termination with respect to $S \subseteq$ 
$\left(U_{M}^{E}\right)^{n}$, if $n>0$ ), if it is non-violating LD-termination (non-violating LDtermination with respect to $S$ ) and non-improving LD-termination (nonimproving LD-termination with respect to $S$ ). The meta-interpreter $M_{0}$ preserves termination and the meta-interpreter $M_{2}$ preserves termination with respect to Vars, that is if it is used to measure the depth of LDrefutation of a give goal, and not to bound it. In the next sections we prove these statements.

\section{Termination of "vanilla" meta-interpreter}

Termination of the "vanilla" meta-interpreter, presented in Example 1, has been studied by Pedreschi and Ruggieri. They proved, that "vanilla" does not violate termination (Corollary 40, [23]). However, we can claim more - this meta-interpreter preserves termination.

We base our proof on soundness and completeness of "vanilla", proved in [19]. Observe that in general soundness and completeness are not sufficient for the calls set to be preserved. Indeed, consider the following example, motivated by the ideas of unfolding [6].

Example 6. The following meta-interpreter $M_{3}$ eliminates calls to undefined predicates.

$$
\begin{aligned}
& \text { solve }(\text { true }) . \\
& \text { solve }((A, B)) \leftarrow \operatorname{solve}(A), \operatorname{solve}(B) . \\
& \operatorname{solve}(A) \leftarrow \operatorname{clause}(A, B), \operatorname{check}(B), \operatorname{solve}(B) . \\
& \operatorname{check}((A, B)) \leftarrow \operatorname{check}(A), \operatorname{check}(B) . \\
& \operatorname{check}(A) \leftarrow \operatorname{clause}(A, \ldots) .
\end{aligned}
$$

This meta-interpreter is sound and complete, i.e., preserves computed answers. However, it does not preserve termination. Indeed, let $P$ be the following program [6]:

$$
p \leftarrow q, r . \quad t \leftarrow r, q . \quad q \leftarrow q .
$$

and let $p$ be the goal. Then, $p$ with respect to $P$ does not terminate, while $\leftarrow \operatorname{solve}(p)$ with respect to $M_{3} \cup \gamma_{c e}(P)$ terminates (finitely fails). Thus, this meta-interpreter does not preserve LD-termination. Observe, that unfolding may only improve termination [6], thus, this meta-interpreter is improving LD-termination. 
Thus, we need some additional result, claiming that the "vanilla" meta-interpreter preserves the calls.

Lemma 1. Let $P$ be an interpreted program, $M_{0}$ be the vanilla metainterpreter and $G \in B_{P}^{E}$, then

$$
\begin{aligned}
& \{\operatorname{solve}(A) \mid A \in \operatorname{Call}(P, G)\} \equiv \\
& \quad \operatorname{Call}\left(M_{0} \cup \gamma_{c e}(P), \operatorname{solve}(G)\right) \cap\left\{\operatorname{solve}(A) \mid A \in B_{P}^{E}\right\}
\end{aligned}
$$

where $\equiv$ means equality up to variable renaming.

Proof. For all proofs we refer to [26].

Theorem 2. Let $P$ be a definite program, $S$ a set of queries, and $M_{0}$ the vanilla meta-interpreter, such that $M_{0} \cup \gamma_{c e}(P)$ is LD-terminating for all queries in $\{\operatorname{solve}(G) \mid G \in S\}$. Then, $P$ is LD-terminating for all queries in $S$.

Proof. (sketch) By Theorem $1 M_{0} \cup \gamma_{c e}(P)$ is order-acceptable with respect to $\{\operatorname{solve}(G) \mid G \in S\}$. We are going to prove order-acceptability of $P$ with respect to $S$. By Theorem 1 this will imply termination.

Since $M_{0} \cup \gamma_{c e}(P)$ is order-acceptable with respect to solve $(S)=$ $\{$ solve $(G) \mid G \in S\}$ there is a well-founded ordering $>$, satisfying requirements of Definition 3. Define a new ordering on atoms of $P$ as following: $A \succ B$ if $\operatorname{solve}(A)>\operatorname{solve}(B)$.

The ordering is defined on $\left\{G \mid \operatorname{solve}(G) \in \operatorname{Call}\left(M_{0} \cup \gamma_{c e}(P)\right.\right.$, solve $\left.\left.(S)\right)\right\}$. By Lemma 1 this set coincides with $\operatorname{Call}(P, S)$. From the corresponding properties of $>$ follows that $\succ$ is well-defined and well-founded.

The only thing that remains to be proved is that $P$ is order-acceptable with respect to $S$ via $\succ$. Let $A \in \operatorname{Call}(P, S)$ and let $A^{\prime} \leftarrow B_{1}, \ldots, B_{n}$ be a clause in $P$, such that $\operatorname{mgu}\left(A, A^{\prime}\right)=\theta$ exists. Then, $\theta$ is also mgu of solve $(A)$ and solve $\left(A^{\prime}\right)$.

Let $\sigma$ map $B \theta$ to $\left(B_{1}, \ldots, B_{n}\right) \theta$. It is one of the computed answer substitutions for $\leftarrow \operatorname{clause}(A \theta, B \theta)$. Thus, by order-acceptability of $M_{0} \cup$ $\gamma_{c e}(P)$ with respect to $\operatorname{solve}(S)$ holds: solve $(A)>\operatorname{solve}\left(\left(B_{1}, \ldots, B_{n}\right) \theta\right)$.

Order-acceptability also implies solve $\left(\left(B_{1}, \ldots, B_{n}\right) \theta\right)>\operatorname{solve}\left(B_{1} \theta\right)$ and solve $\left(\left(B_{1}, \ldots, B_{n}\right) \theta\right)>\operatorname{solve}\left(\left(B_{2}, \ldots, B_{n}\right) \theta \sigma_{1}\right)$, where $\sigma_{1}$ is a computed answer substitution for solve $\left(B_{1} \theta\right)$. By proceeding in this way we conclude, that for any atom $B_{i}$, solve $(A)>\operatorname{solve}\left(B_{i} \theta \sigma_{1} \ldots \sigma_{i-1}\right)$, where $\sigma_{j}$ is a computed answer substitution for $\leftarrow \operatorname{solve}\left(B_{j} \theta \sigma_{1} \ldots \sigma_{j-1}\right)$. By definition of $\succ$, this means that $A \succ B_{i} \theta \sigma_{1} \ldots \sigma_{i-1}$.

Soundness and completeness imply the order-acceptability and complete the proof. 
The second direction of the theorem has been proved by Pedreschi and Ruggieri [23]. It allows us to state the following corollary.

Corollary 1. Tha "vanilla" meta-interpreter preserves LD-termination.

The proof of Theorem 2 sketched above, suggests the following methodology for proving that some meta-interpreter improves LD-termination. First, define an ordering on the set of calls to the meta-interpreter, that reflects its behaviour. Then, establish the relationship between a new ordering and the one that reflects order-acceptability with respect to a set of the interpreted program. Prove using this relationship that the newly defined ordering is well-defined, well-founded and reflects orderacceptability of the meta-program with respect to a corresponding set of calls. In order for the proofs to be correct one may need to assume (or to prove as a prerequisite) that the meta-interpreter is sound and that the set of calls of the interpreted program and of the meta-program correspond to each other. The opposite direction can be proved by using a similar methodology.

\section{Advanced meta-interpreters}

Typical applications of meta-interpreters, such as debuggers, will also require producing some additional output or performing some additional tasks during the execution, such as constructing proof trees or cutting "unlikely" branches for an uncertainty reasoner with cutoff. As we are going to see in Example 7 these extensions can and usually will influence termination properties of the meta-interpreter.

In this section we identify an important class of meta-interpreters that are able to perform additional tasks and extend the methodology presented in the previous section to analyse them.

Definition 10. A definite program of the following form

$$
\begin{aligned}
& \operatorname{solve}\left(\operatorname{true}, t_{11}, \ldots, t_{1 n}\right) \leftarrow C_{11}, \ldots, C_{1 m_{1}} . \\
& \operatorname{solve}\left((A, B), t_{21}, \ldots, t_{2 n}\right) \leftarrow \\
& \quad D_{11}, \ldots, D_{1 k_{1}}, \operatorname{solve}\left(A, t_{31}, \ldots, t_{3 n}\right), \\
& \quad D_{21}, \ldots, D_{2 k_{2}}, \operatorname{solve}\left(B, t_{41}, \ldots, t_{4 n}\right) \\
& \quad C_{21}, \ldots, C_{2 m_{2}} . \\
& \operatorname{solve}\left(A, t_{51}, \ldots, t_{5 n}\right) \leftarrow \\
& \quad D_{31}, \ldots, D_{3 k_{3}}, \operatorname{clause}\left(A, B, s_{1}, \ldots, s_{k}\right),
\end{aligned}
$$




$$
\begin{aligned}
& D_{41}, \ldots, D_{4 k_{4}}, \text { solve }\left(B, t_{61}, \ldots, t_{6 n}\right) \\
& C_{31}, \ldots, C_{3 m_{3}} .
\end{aligned}
$$

together with defining clauses for any other predicates occurring in the $C_{k l}$ and $D_{p q}$ (none of which contain solve or clause) is called a double extended meta-interpreter.

This class of meta-interpreters extends the class of extended metainterpreters studied by [22]. It includes many useful meta-interpreters, such as a proof trees constructing meta-interpreter [28], that can be used as a basis for explanation facilities in expert system, meta-interpreters allowing reasoning about theories and provability $[8,21]$ or reasoning with uncertainty [28]. Moreover, this class also describes a depth tracking tracer for Prolog, a reasoner with threshold cutoff [28] and a pure four port box execution model tracer [7]. Note, that despite the similarity with Example 6, Example 6 is not a double extended meta-interpreter due to the call to predicate clause in the definition of check.

Definition 11. Let $H \leftarrow B_{1}, \ldots, B_{i}, \ldots, B_{n}$ be a clause, and let $v$ be a variable in $B_{i}$. We'll say that $\leftarrow B_{1}, \ldots, B_{i-1}$ is irrelevant for $v$ if for every computed answer substitution $\sigma$ for $\leftarrow B_{1}, \ldots, B_{i-1}, v \sigma=v$.

We'll call a double extended meta-interpreter restricted if for any $i$, $\leftarrow D_{i 1}, \ldots, D_{i k_{i}}$ is irrelevant for meta-variables $A$ and $B$. The following theorem states conditions on the restricted double extended metainterpreter $E$ that imply $E$ to be non-violating LD-termination.

Theorem 3. Let $P$ be an interpreted program, $D$ a restricted double extended meta-interpreter, and $G \in B_{D \cup \gamma_{c e}(P)}^{E}$, such that $G$ is terminating with respect to $P$ and $\left\{A \mid A \in \operatorname{Call}\left(D \cup \gamma_{c e}(P)\right.\right.$, solve $\left.(G)\right)$, solve $\left.\sqsupset \operatorname{rel}(A)\right\}$ is terminating with respect to $D$ then solve $(G)$ terminates with respect to $D \cup \gamma_{c e}(P)$.

In general, the second direction of the theorem does not necessary hold. Indeed, consider the following uncertainty reasoner with cutoff, motivated by [28].

Example $\%$.

$$
\begin{array}{cl}
\text { solve }(\text { true }, 1, T) . & \operatorname{solve}(A, C, T) \leftarrow \\
\text { solve }((A, B), C, T) \leftarrow & \text { clause }(A, B, C 1), \\
\text { solve }(A, C 1, T), & C 1>T, T 1 \text { is } T / C 1, \\
\text { solve }(B, C 2, T), & \text { solve }(B, C 2, T 1), \\
\text { minimum }(C 1, C 2, C) . & C \text { is } C 1 * C 2 .
\end{array}
$$

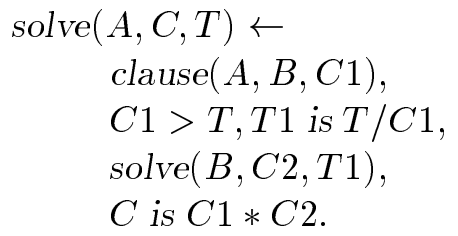


Let $P$ be the following uncertainty-program: clause $(r, r, 0.9)$. When executed as a usual program, ignoring uncertainty coefficients, the goal $\leftarrow r$ does not terminate. However, for any specified threshold Threshold $>0$ the goal $\leftarrow$ solve $(r$, Certainty, Threshold $)$ finitely fails.

Termination of this example followed from the fact that given some positive threshold Threshold there always be a natural $n$, such that $0.9^{n}<$ Threshold. To cause the second direction of Theorem 3 to hold this kind of termination should be eliminated. That is, termination of the meta-program shall depend only on the meta-calls. To formalise this intuition we set a requirement on the ordering used to prove termination of the meta-program. We say that an ordering $>$ ignores argument position $i$ of a functor $f$, if for all $t_{1}, \ldots, t_{n}$, for all $s_{1}, s_{2}$ and for all $u$, if $f\left(t_{1}, \ldots, t_{i-1}, s_{1}, t_{i+1}, \ldots, t_{n}\right) \rho u$ then $f\left(t_{1}, \ldots, t_{i-1}, s_{2}, t_{i+1}, \ldots, t_{n}\right) \rho u$, for every $\rho \in\{<,>,=, \|\}$.

Theorem 4. Let $P$ be an interpreted program and $D$ a double extended meta-interpreter, such that $D \cup \gamma_{c e}(P)$ is order-acceptable with respect to solve $(G)$ via an ordering $>$, that ignores all argument positions of solve, except for the first one. Then,

1. D terminates w.r.t. $\left\{A \mid A \in \operatorname{Call}\left(D \cup \gamma_{c e}(P)\right.\right.$, solve $\left.(G)\right)$, solve $\sqsupset$ $\operatorname{rel}(A)\}$ and

2. $P$ terminates w.r.t. $G$.

\section{Extending the language of the interpreted programs}

So far we have considered only definite programs. However, in order to make our approach practical the language of the underlying object programs should be extended to include negation, frequently appearing in applications of the meta-interpreters.

In order to prove that meta-interpreters with negation preserve termination we use among others a termination analysis framework based on order-acceptability. Originally this framework was designed only for study of termination of definite programs. In [26] we extended this framework to normal programs as well. Here we present briefly some results that can be obtained for the meta-interpreters in the extended framework.

First of all, instead of LD-derivations and trees LDNF-derivations and trees should be considered. Recall, that LDNF-derivation flounders if there occurs in it or in any of its subsidiary LDNF-trees a goal with the first literal being non-ground and negative. 
Definition 12. The program $P$ is called non-floundering with respect to a set of queries $S$, if all its $L D N F$-derivations starting in queries $G \in S$ are non-floundering.

By extending the notion of order-acceptability to normal programs and applying the same methodology as above one can prove that the following meta-interpreter $M_{4}$, being an immediate extension of vanilla meta-interpreter to normal programs [16], preserves LDNF-termination. Soundness and completeness of $M_{4}$ are proved in Theorem 2.3.3 [17].

$$
\begin{aligned}
& \text { solve }(\text { true }) . \\
& \text { solve }((\text { Atom, Atoms })) \leftarrow \text { solve }(\text { Atom }), \text { solve }(\text { Atoms }) . \\
& \text { solve }(\neg \text { Atom }) \leftarrow \neg \text { solve }(\text { Atom }) . \\
& \text { solve }(\text { Head }) \leftarrow \text { clause }(\text { Head, Body }), \text { solve }(\text { Body }) .
\end{aligned}
$$

Theorem 5. Let $P$ be a normal program, $S$ be a set of queries. Then $P$ is LDNF-terminating w.r.t. $S$ if and only if $M_{4} \cup \gamma_{c e}\left(P^{\prime}\right)$ LDNF-terminates with respect to $\{\operatorname{solve}(G) \mid G \in S\}$.

\section{Conclusion}

We have presented a methodology for proving termination properties of meta-programs. The problem of termination was studied by a number of authors (see [12] for the survey, and more recent work can be found in [13, $14,18,20,24,27,30])$.

Our methodology gains it power from using the integrated approach, suggested in [25], that extends the traditional notion of acceptability [2] with the wide class of term-orderings that have been studied in the context of the term-rewriting systems. In this work we have shown that this approach allows one to define relatively simple relation between the ordering that satisfies the requirements of order-acceptability for an object program and for the meta-interpreter extended by this object program and a corresponding set of goals. Thus, the methodology is useful to establish if the meta-interpreter improves or preserves termination. In particular, in the work on compositionality of termination proofs [3], level mappings for an object program cannot easily be reused for the meta-program.

Despite the intensive research on meta-programming inside the logic programming community $[4,19,22]$ termination behaviour of metaprograms attracted less attention. Pedreschi and Ruggieri [23] use generic verification method, based on specifying preconditions and postconditions. Unfortunately, their termination results are restricted only to the 
"vanilla" meta-interpreter. It is not immediate how their results can be extended to alternative meta-interpreters, nor if the relationship between termination characterisation of the object program and the meta-program can be established.

We consider as a future work identifying additional classes of metainterpreters, such as $[9,11,29]$ and studying their termination behaviour.

\section{Acknowledgement}

Alexander Serebrenik is supported by GOA: " $L P^{+}$: a second generation logic programming language".

\section{References}

1. K. R. Apt. From Logic Programming to Prolog. Prentice-Hall Int. Series in Computer Science. Prentice Hall, 1997.

2. K. R. Apt and D. Pedreschi. Studies in Pure Prolog: Termination. In J. W. Lloyd, editor, Proc. Esprit Symp. on Comp. Logic, pages 150-176. Springer Verlag, 1990.

3. K. R. Apt and D. Pedreschi. Modular termination proofs for logic and pure prolog programs. In G. Levi, editor, Advances in Logic Programming Theory, pages 183229. Oxford University Press, 1994.

4. K. R. Apt and F. Turini, editors. Meta-Logics and Logic Programming. Logic Programming. The MIT Press, 1995.

5. T. Arts. Automatically proving termination and innermost normalisation of term rewriting systems. PhD thesis, Universiteit Utrecht, 1997.

6. A. Bossi and N. Cocco. Preserving universal temination through unfold/fold. In G. Levi and M. Rodríguez-Artalejo, editors, Algebraic and Logic Programming, pages 269-286. Springer Verlag, 1994. LNCS 850.

7. A. Bowles and P. Wilk. Tracing requirements for multi-layered meta-programming. In H. Abramson and M. H. Rogers, editors, Meta-Programming in Logic Programming, pages 205-216. The MIT Press, 1989.

8. A. Brogi, P. Mancarella, D. Pedreschi, and F. Turini. Composition operators for logic theories. In J. W. Lloyd, editor, Proc. Esprit Symp. on Comp. Logic, pages 117-134. Springer Verlag, 1990.

9. M. Bruynooghe, D. De Schreye, and B. Martens. A general criterion for avoiding infinite unfolding during partial deduction. New Generation Computing, 11(1):4779, 1992.

10. F. Bueno, M. J. García de la Banda, and M. V. Hermenegildo. Effectiveness of global analysis in strict independence-based automatic parallelization. In M. Bruynooghe, editor, Logic Programming, Proc. of the 1994 Int. Symp., pages 320-336. MIT Press, 1994.

11. M. H. Cheng, M. H. van Emden, and P. A. Strooper. Complete sets of frontiers in logic-based program transformation. In H. Abramson and M. H. Rogers, editors, Meta-Programming in Logic Programming, pages 283-297. The MIT Press, 1989.

12. D. De Schreye and S. Decorte. Termination of logic programs: The never-ending story. J. Logic Programming, 19/20:199-260, May/July 1994. 
13. S. Decorte and D. De Schreye. Termination analysis: some practical properties of the norm and level mapping space. In J. Jaffar, editor, Proc. of the 1998 Joint Int. Conf. and Symp. on Logic Programming, pages 235-249. MIT Press, June 1998.

14. S. Decorte, D. De Schreye, and H. Vandecasteele. Constraint-based termination analysis of logic programs. ACM Transactions on Programming Languages and Systems (TOPLAS), 21(6):1137-1195, November 1999.

15. N. Dershowitz and Z. Manna. Proving termination with multiset orderings. Communications of the ACM (CACM), 22(8):465-476, August 1979.

16. P. Hill and J. Gallagher. Meta-programming in logic programming. In D. M. Gabbay, C. Hogger, and J. Robinson, editors, Handbook of logic in Artificial Intelligence and Logic Programming, pages 421-498. Clarendon press, 1998. volume 5. Logic Programming.

17. P. Hill and J. Lloyd. Analysis of meta-programs. In H. Abramson and M. H. Rogers, editors, Meta-Programming in Logic Programming, pages 23-52. The MIT Press, 1989.

18. S. Hoarau. Inférer et compiler la terminaison des programmes logiques avec contraintes. PhD thesis, Université de La Réunion, 1999.

19. G. Levi and D. Ramundo. A formalization of metaprogramming for real. In D. S. Warren, editor, Logic Programming, Proceedings of the Tenth International Conference on Logic Programming, pages 354-373. MIT Press, 1993.

20. N. Lindenstrauss and Y. Sagiv. Automatic termination analysis of logic programs. In L. Naish, editor, Proc. of the Fourteenth Int. Conf. on Logic Programming, pages 63-77. MIT Press, July 1997.

21. B. Martens and D. De Schreye. Two semantics for definite meta-programs, using the non-ground representation. In K. Apt and F. Turini, editors, Meta-Logics and Logic Programming, pages 57-81. MIT Press, Cambridge, MA, 1995. ISBN: 0-262-01152-2.

22. B. Martens and D. De Schreye. Why untyped nonground metaprogramming is not (much of) a problem. Journal of Logic Programming, 22(1):47-99, January 1995.

23. D. Pedreschi and S. Ruggieri. Verification of meta-interpreters. Journal of Logic and Computation, 7(2):267-303, November 1997.

24. S. Ruggieri. Verification and validation of logic programs. PhD thesis, Universitá di Pisa, 1999

25. A. Serebrenik and D. De Schreye. Non-transformational termination analysis of logic programs, based on general term-orderings. In K.-K. Lau, editor, Logic Based Program Synthesis and Transformation 10th International Workshop, Selected Papers, volume 2042 of Lecture Notes in Computer Science, pages 69-85. Springer Verlag, 2001.

26. A. Serebrenik and D. De Schreye. On termination of metaprograms. Technical Report CW 306, Departement Computerwetenschappen, K.U.Leuven, Leuven, Belgium, 2001. Available at http://www.cs.kuleuven.ac.be/publicaties/rapporten/CW2001.html.

27. J.-G. Smaus. Modes and Types in Logic Programming. PhD thesis, University of Kent, 1999.

28. L. Sterling and E. Shapiro. The Art of Prolog. The MIT Press, 1994.

29. F. van Harmelen. A classification of meta-level architectures. In H. Abramson and M. H. Rogers, editors, Meta-Programming in Logic Programming, pages 103-122. The MIT Press, 1989.

30. S. Verbaeten. Static verification of compositionality and termination for logic programming languages. PhD thesis, Department of Computer Science, K.U.Leuven, Leuven, Belgium, June 2000. v+265+xxvii. 